\title{
The thoracic duct: Predictably unpredictable?
}

\author{
Jonathan M. Chen, MD
}

\author{
From Congenital Cardiac Surgery, Seattle Children's Hospital, and Department of Surgery, University of Wash- \\ ington School of Medicine, Seattle, Wash. \\ Disclosures: Author has nothing to disclose with regard to commercial support. \\ Received for publication July 4, 2015; accepted for publication July 6, 2015; available ahead of print July 30, \\ 2015. \\ Address for reprints: Jonathan M. Chen, MD, Seattle Children's Hospital, 4800 Sand Point Way NE, RC 2.820, \\ Seattle, WA 98105 (E-mail: jmchen@uw.edu). \\ J Thorac Cardiovasc Surg 2015;150:497 \\ $0022-5223 / \$ 36.00$ \\ Copyright (C) 2015 by The American Association for Thoracic Surgery \\ http://dx.doi.org/10.1016/j.jtcvs.2015.07.013
}

Among the litany of seemingly mundane potential complications of cardiac surgery, chylothorax remains one of the most vexing, the contributions of which to overall morbidity should not be underestimated, in particular for pediatric patients. In this issue of the Journal, Bang and colleagues ${ }^{1}$ present their 22-year experience with management of chylothorax, utilizing a primary approach of right thoracotomy/mass ligation in the vast majority, and additional approach via left thoracotomy in 10 patients with ongoing drainage. ${ }^{1}$ Although the presence of dextrocardia appeared to be more common in the 10 patients requiring additional left thoracotomy, the traditional characteristics of heterotaxy and complex congenital heart disease (eg, atrial situs and bilateral cavae) did not. Moreover, unlike other such investigations in the literature, this was not a study that followed specific clinical practice guidelines, and insofar as this is true, its conclusions are somewhat limited. ${ }^{2,3}$

As the authors suggest, the course of the thoracic duct is notoriously variable, and indeed a recent literature review suggested that the thoracic duct may have multiple channels in $40 \%$ of patients. ${ }^{4}$ Their approach involving additional left thoracotomy, however, was not driven by advanced imaging directing additional intervention, but rather by empiric ongoing drainage despite attempted mass ligation from the right side. Investigations into additional causes of ongoing drainage (eg, venous thrombosis) were not routinely pursued.

What, then, can we infer from this study? Certainly it appeals to reason that earlier laboratory diagnosis, and by extension perhaps earlier surgical intervention, could reduce the number of days of ongoing drainage, fluid imbalance, and inadequate nutrition, not to mention overall cost. Yet this, too, would be speculative. Waiting 1 more day to evaluate the trend in drainage always seems appealing, and for those who ultimately evade a second operation, may appear less injurious. To be sure, in the realm of postoperative chylothorax, there are no atheists in foxholes: When the chylous chips are down, we are willing to put our faith into virtually anything - adjunctive medical therapies (eg, octreotide); alternative operations (eg, ligation of the cysterna); and adjunctive procedures before, during, and after mass ligation (in this case $26 \%$ received

\section{References} Surg. 2005;80:1864-71.

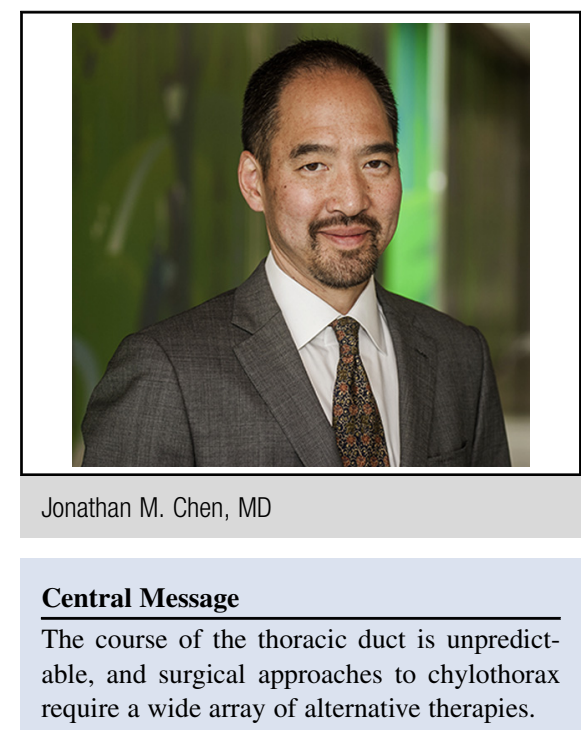

See Article page 490.

pleurodesis) - to address the problem if the ultimate result is recovery, chest tube removal, and home discharge.

However, the results of these varied approaches are potentially as unpredictable as the anatomic course of the thoracic duct itself. Having an armamentarium of therapeutic approaches for the management of chylothorax is undeniably advantageous, although the timing and order of implementation (despite the current study) remains elusive. A high index of suspicion for unusual anatomy seems prudent, potentially (as these authors suggest) for those patients with dextrocardia, and dogged persistence in the pursuit of the elusive thoracic duct is mandatory in the case of recalcitrant chylothorax. Although this knowledge is unlikely to change our initial cardiac reparative techniques, it may provide an additional detail as we attempt to refine ongoing clinical pathway recommendations.

1. Bang JH, Kim SH, Park CS, Park J, Yun T. Anatomic variability of the thoracic duct in pediatric patients with complex congenital heart disease. J Thorac Cardiovasc Surg. 2015;150:490-6.

2. Yeh J, Brown ER, Kellogg KA, Donohue JE, Yu S, Gaies MG, et al. Utility of a clinical practice guideline in treatment of chylothorax in the postoperative congenital heart patient. Ann Thorac Surg. 2013;96:930-7.

3. Chan EH, Russell JL, Williams WG, Van Arsdell GS, Coles JG, McCrindle BW Postoperative chylothorax after cardiothoracic surgery in children. Ann Thorac

4. Phang K, Bowman M, Phillips A, Windsor J. Review of thoracic duct anatomical variations and clinical implications. Clin Anat. 2014;27:637-44.

5. Zanin A, Padalino MA, Cerutti A, Vida VL, Milanesi O, Stellin G, et al. Surgical ligation of the cysterna chili: an alternative treatment for chronic chylothorax in children. Ann Thorac Surg. 2010;90:1732-4. 